\title{
Flow kinematics with oppositely rotating coaxial layers
}

\author{
Genrikh Orekhov* \\ Moscow State University of Civil Engineering, Yaroslavskoe shosse, 26, Moscow, 129337, Russia
}

\begin{abstract}
In modern technological processes, liquid and gas flows play an important role. This is especially mostly evident in hydraulic engineering and hydropower construction when designing the highly efficient hydraulic turbines, various spillway systems and other elements of layout patterns of structures. When designing and constructing such facilities, it is necessary to know the characteristics of the flows to be passed through these structures in order to correctly take into account the various types of impacts from the flow: pressure pulsations, cavitation and erosion due to cavitation, wave formation and abrasive wear. When designing high-head spillway structures, special attention is paid to the presence of excess energy from the stream entering the lower pool, which requires special measures to dissipate it. Nowadays, in the practice of construction of hydraulic structures, swirled flows are used including those with oppositely rotating layers. This type of flow creates a number of effects that allow one to radically solve the problems of energy dissipation of high-velocity flows. The report presents the results of studies of a complicated flow formed by oppositely rotating coaxially arranged layers of liquid made by a physical method. The description of the experimental stand for the model studies of such flows is given. The basic parameters of the installation, the principle of operation of the recording equipment and its main characteristics are given. The results of model studies in the form of distribution of the components of the flow velocity along the cross section of the flow conductor and its length are given.
\end{abstract}

\section{Introduction}

Swirling streams of liquid and gas have found their application in technological processes used in various devices, installations and structures. Their wide spread is associated with a number of important properties inherent to such flows compared with conventional axial flows. Swirled flows are used in processes that ensure atomization of liquid fuel [1,2], mixing [3] and dispersion of fluids, forming aerosols, flames [4] and other technological processes. On the basis of deep and extensive studies of swirling flows, the devices have been started to be created that make it possible to repeatedly intensify the processes of energy, mass and heat exchange in heat engineering, hydraulic machine engineering, aircraft building, mechanical engineering and in a number of other industries [5, 6]. Structures, installa-

\footnotetext{
*Corresponding author: orehov_genrih@mail.ru
} 
tions and apparatus, whose work is based on the use of effects of swirling streams, are called vortex devices.

Along with developments in other areas, researchers conducted numerous experiments to determine various hydraulic and hydrodynamic characteristics of swirling flows for their use in hydraulic engineering, hydro-amelioration, hydro-mechanical construction, hydrotransport $[7,8,9,10,11]$. One of the most interesting directions in studying the behavior of swirling flows was the study of interacting swirling flows of liquid and gas with coaxially arranged layers $[12,13]$.

Suggestions for the use of interacting flows were prompted by practice requirements. This is primarily the flow energy dissipation in high-head hydraulic systems, mixing of two-phase flows consisting of liquids and gases $[14,15]$. The method of flow energy dissipation using the interaction of flows (jets) is based on the idea of dividing the initial flow into parts and creating conditions for the mutual energy dissipation of individual flow parts with their subsequent connection. As known, the water flow moving from the upper pool of a hydraulic structure downstream dissipates its energy either by performing useful work when installing in the flow a device that draws this energy, or by overcoming the forces that necessarily occur when the flow passes through various structures. The energy of one part of the flow when interacting with the energy of the other part is used to create forces equivalent to the reactive forces developed by dissipators. Such interaction can give the maximum effect of dissipation of the initial flow energy in the conditions of a rational splitting of the flow into parts and creating the proper directions of movement of these parts relative to each other. The nature and intensity of hydrodynamic processes occurring in such devices ensure the effectiveness of their application in a wide variety of modern technologies to achieve different technological goals.

Fluid or gas flows representing the flow of interacting oppositely rotating coaxial layers are extremely complicated spatial flows with hydraulic characteristics that differ significantly from conventional longitudinal-axial flows $[16,17]$. This article is devoted to the results of model studies of such flows with different number of interacting layers, which may be of particular interest in various technical and technological applications.

\section{Objective}

The purpose of this work is to conduct studies of a complicated flow formed by oppositely rotating axial fluid layers by the methods of physical modeling using currently available laser technology for measuring flow parameters. The method of tracer visualization of the flow with the help of PIV equipment allowing for recording the flow parameters in a threedimensional representation is used as the main measuring method.

The researches were aimed to obtain the distribution of velocity components in the area of interaction of oppositely swirled layers, vector fields and streamlines.

\section{Method}

Liquid or gas flows with oppositely rotating coaxial layers structurally are complicated flows. They include recirculation zones with velocity fields inverse to the general direction of flow formed within these zones. The entire flow in the zone of interaction of the layers has a high value of turbulence and a cellular cascade structure of vortex fields. Therefore, studies of such flows are performed in two ways: by mathematical and physical modeling. Both methods, being approximate methods for solving problems of hydro and aerodynamics, complement and correct each other. 
To determine the main kinematic characteristics of circulating interacting flows, experiments were carried out on models using laser technology for contactless determination of flow parameters - the tracer flow visualization in 3D modification.

Figure 1 shows the flow conductor part of the model with PIV measuring system. The longitudinal-axial flow moving with $v$ velocity along the circular cylindrical channel enters the system of swirler vane row 1 breaking the initial flow into several coaxial layers, swirled

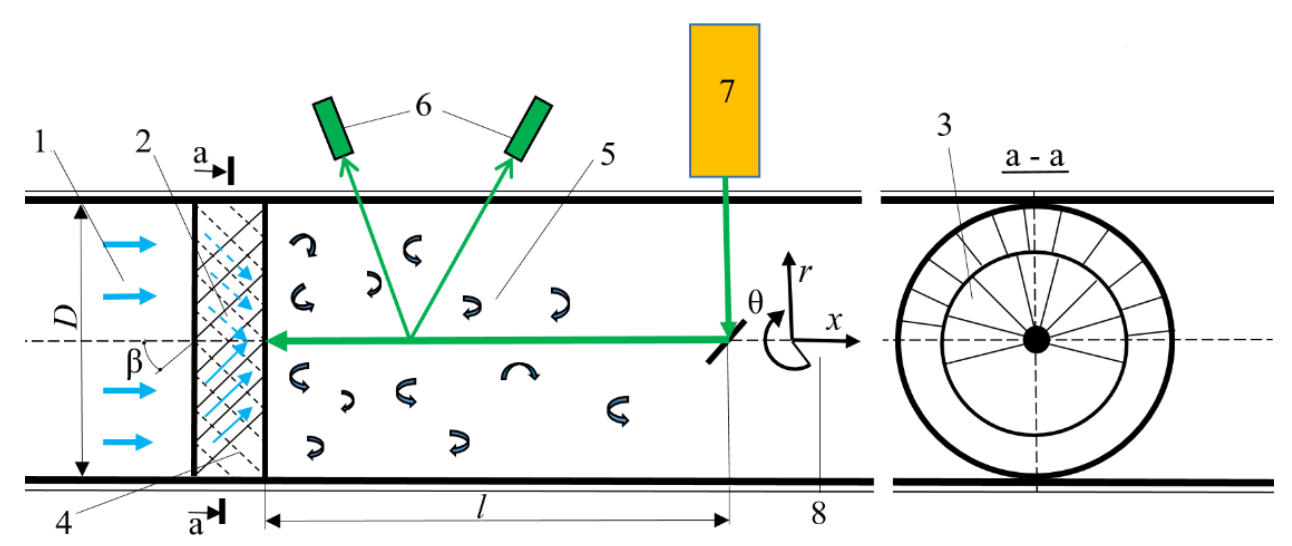

Fig. 1. Model experimental installation. Schematic flow organization of oppositely rotating coaxial layers using swirlers of axial blade type and forming 3D laser fixation of flow parameters. 1 - circular cylindrical flow conductor part of the model, 2 - axial vane swirler of the flow layers, 3 - axial vane swirler, view by $a-a, 4$ - swirler vanes installed at an angle $\alpha$ to the axis of the flow conductor, 5 interaction area of coaxial flows of $l$ length formed by oppositely rotating layers, 6 - velocity matrix chambers of process fixation, 7 - laser emitter forming illumination of tracer particles in the flow area under study, $8-x, r, \theta-$ axes of the considered cylindrical coordinate system

in opposite directions. In the experiment three models of swirlers have been used, each of which forming flows with two, four, and six oppositely rotating coaxial layers. The first swirler model is double-layered - the vanes of the peripheral row twist a part of the incident flow (layer) clockwise, the vanes of the inner row twist the remaining part of the incoming flow counterclockwise. At the same time, two oppositely twisted coaxially located flows begin to interact behind the local swirler in the mixing chamber. The second model is four layered. Here four coaxially arranged and twisted in pairs in opposite directions layers are formed. The third model is six-layered and has six vane rows.. This model forms six layers coaxially arranged and pairwise twisted in opposite directions. The models are made of plastic on a 3D printer. The outer diameter of all the swirlers is the same and is $200 \mathrm{~mm}$ (equal to the diameter of the flow conductor pipe). The photo of swirler models is shown in Fig. 2, $a$.

The flow area under study was recorded by two chambers 6 . The illumination was performed by a high-power pulsed laser. The photo of the experimental installation at the moment of illumination with a pulsed laser is shown in Fig. 2, $b$.

When studying the flows with the aid of the PIV system of importance is the qualitative sowing the flow under study with tracers. Geometric dimensions of the particles, their physical properties, as well as the adequate concentration in the flow area under study, are the necessary conditions for the efficient operation of the entire measuring system. Special particles previously placed into the liquid or gas flow serve as velocity sensors. The particles must be small enough to follow up without distortion the streamlines of the flow under 
study. On the other hand, the particles must be large enough to reflect the light in the quantity necessary for its recording with digital cameras 6 (see Fig. 1). In the ideal case, the particles should have a neutral buoyancy, that is, have a density equal to the density of the liquid or gas composing the flow under study. Particle sizes in the experiments using PIV complexes can vary from 100 to $50,000 \mathrm{~nm}$ (nanometers). Such dimensions are comparable with the laser radiation wavelength $(532 \mathrm{~nm})$ and, hence, subject to the conditions of light scattering Mie. In the experiments conducted, a special liquid (synthetic oil) has been used. The installation, which includes a compressor and nozzles, sprays liquid to a particle size of approximately $1000 \mathrm{~nm}$. An important characteristic of tracer particles is their settling velocity, the velocity with which the particles sink down under the effect of gravity. This parameter is especially important when studying the low-velocity flows.

The average flow rate velocity $V$ of the flow under study was determined as

$$
V=Q / F \text {, }
$$

where, $Q$ - is the air flow rate, $F$ is the cross-sectional area of the chamber of interaction of the layers $0.2 \mathrm{~m}$ in diameter. In our experiments, the average flow rate was $2.85 \mathrm{~m} / \mathrm{s}$. The Reynolds number is $R e=3.8 \times 10^{4}$. It is calculated by the average velocity and diameter $D$ of the interaction chamber of the layers (see Fig. 1).
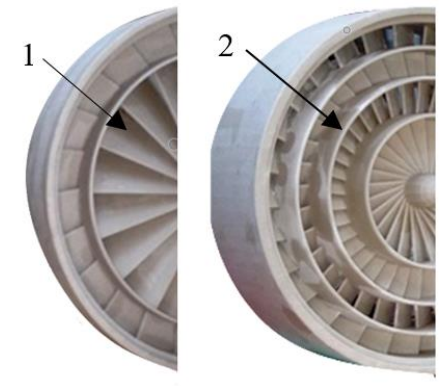

$a$

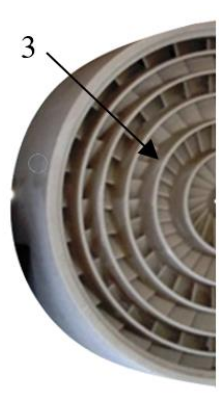

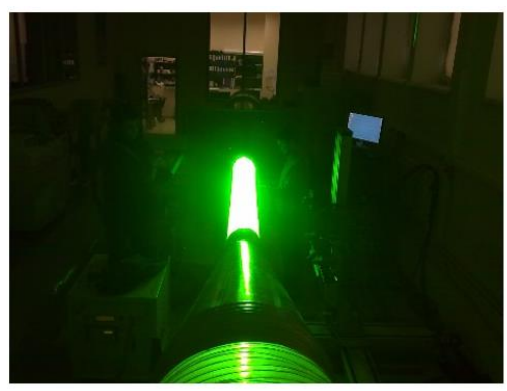

$b$

Fig. 2. $a$ - photos of the swirlers: 1 - two-layered (two blade grids), 2 - four-layered (four blade grids), 3 - six-layered (six blade grids); $b$ - flow conductor of the model installation during the operation of a pulsed illumination laser

\section{Results}

The basic principle applied in the design of the swirlers is the equality of the moments of the amount of movement of the longitudinally circulating layers rotating in opposite directions.

To ensure that at the exit from the interaction area of oppositely rotating layers of length $l$ (Fig. 1), the total flow was free from residual circulation, it must be defined in a certain way at the entrance to it. This is possible under the condition when interacting oppositely swirled layers would have moment of momentum mutually compensating each other. That is, the total moment of momentum of the layers of one direction of rotation must be compensated for by the summary moment of momentum of opposite rotation equal to it.

Then, assuming that the direction of rotation is determined by the sign of the moment, we can write the equality

$$
\sum_{i=1}^{n} M_{i}=0
$$

where: $M_{i}-$ moment of momentum, $n$-number of interacting layers. 
Thus, with the two-layered flow equality $M_{1}=M_{2}$, must be observed, with the fourlayered flow $M_{1}+M_{3}=M_{2}+M_{4}$ equality and with the six-layered flow equality $M_{1}+M_{3}+M_{5}=M_{2}+M_{4}+M_{6}$ must be observed.

The moment of the momentum with index "1" corresponds to the swirled layer formed by the peripheral vane row coupled with the pipe wall. Accordingly, the moment of momentum with index " 6 " corresponds to the layer formed by the innermost vane row associated with the central body of rotation. The remaining indices correspond to the layers that are intermediate between " 1 " and " 6 ".

The moment of momentum of the swirled layer is equal to

$$
M=2 A I R_{\Gamma}=2 A \rho Q V R_{\Gamma}
$$

where: $A$ - dimensionless geometric characteristics of the swirler, $\rho$ - medium density, $Q$ discharge, $V$ - average flow rate, $R_{\Gamma}$ - hydraulic radius, $I$ - the moment of momentum of the circulation-longitudinal flow.

The models used vane rows with a constant angle of installation with respect to the axis. With the vane row setting angle unchanged along the radius, the geometric swirl parameter is characterized by expression

$$
A=\frac{\pi R_{1} R_{0}}{\Omega} \sin \beta
$$

where: $R_{1}$ - outer radius of the vane row (the point of attachment of the vane to the pipe wall or the inner surface of the intermediate separation rim), $R_{0}$ is the effective radius, $\Omega$ is the area of the culvert section between the vane rows, normal to the velocity vector of the descending flow, $\beta$ is the angle of installation of the vane of the vane row in question, that is, the angle between the skeleton line of the vane and the axial line of the swirler (in our case, the axial line of the vane swirler coincides with the axial line of the entire flow conductor of the model).

The effective radius $R_{0}$ and area $\Omega$ are calculated by formulas

$$
R_{0}=\sqrt{\frac{R_{1}^{2}+R_{2}^{2}}{2}} \text { и } \Omega=\pi\left(R_{1}^{2}-R_{2}^{2}\right) \cos \beta .
$$

In (5) $R_{2}$ is the inner radius of the vane row (the point of attachment of the vane to the outer surface of the intermediate separation rim).

The results of studies of the considered flows formed by various swirlers are shown in the form of experimental distributions of the flow velocity components in the cross sections of interaction area $l$ of the layers. In a general case, the movement of a fluid is characterized, along with the azimuthal $u_{\theta}$ also by axial $u_{x}$ and radial $u_{r}$ velocities. The full velocity in an arbitrary point of flow will be equal to

$$
U=\sqrt{u_{r}^{2}+u_{\theta}^{2}+u_{x}^{2}}
$$

Figure 3-5 shows the experimental profiles of axial and azimuthal velocities for two-, four-, and six-layered flows. Measuring sections were located following downstream distant from the swirlers: 20.8, 125, 229, 437, 645, 1180 and $2050 \mathrm{~mm}$ respectively.

It can be seen that in the sections close to the beginning of the interaction area of the layers in the paraxial zones, there are reverse flows with significant negative velocities. This is observed in all three flows under study. This zone is most clearly manifested in the two-layered flow (Fig. 3). It should be noted that the peripheral velocities for the two measuring sections that are closest to the swirling device significantly exceed the average flow rate calculated by (1), 


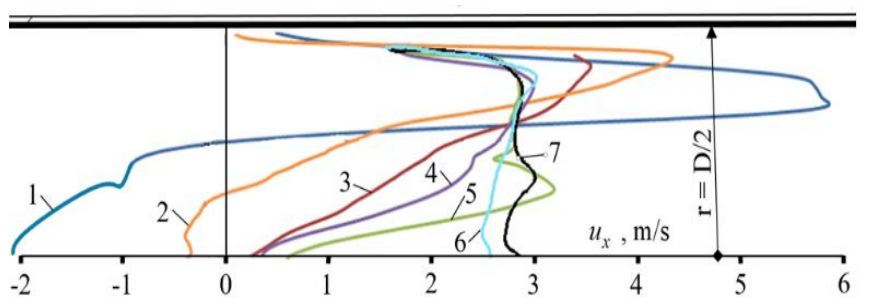

$a$

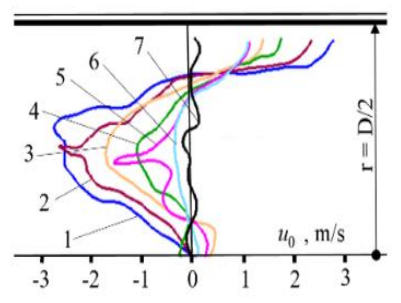

$b$

Fig. 3. Two-layered flow. Profiles: $a-$ axial $u_{x}$ and $b$ - azimuthal $u_{\theta}$ flow velocities. $1-0.2 r, 2-$ $1.3 r, 3-2.3 r, 4-4.4 r, 5-6.6 r, 6-11.3 r, 7-18.2 r$

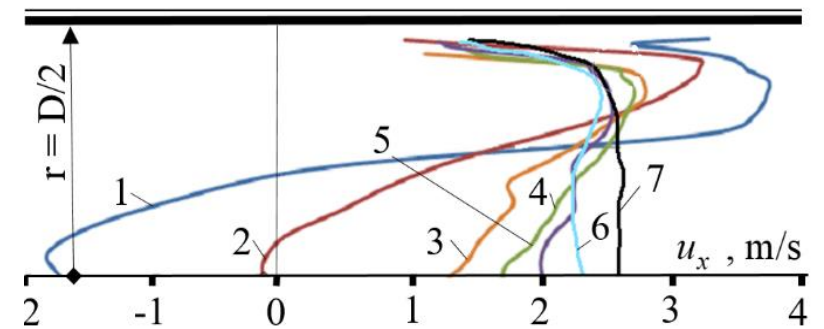

$a$

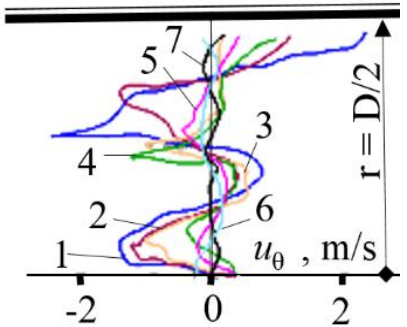

$b$

Fig. 4. Four-layered flow. Profiles: $a-$ axial $u_{x}$ and $b$ - azimuthal $u_{\theta}$ flow velocities. $1-0.2 r, 2-$ $1.3 r, 3-2.3 r, 4-4.4 r, 5-5.9 r, 6-9.7 r, 7-13.1 r$

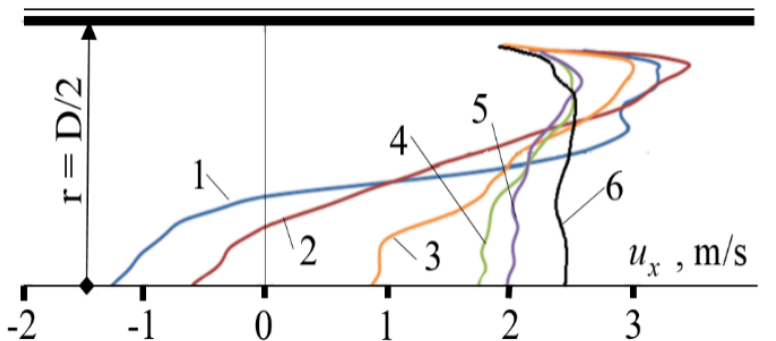

$a$

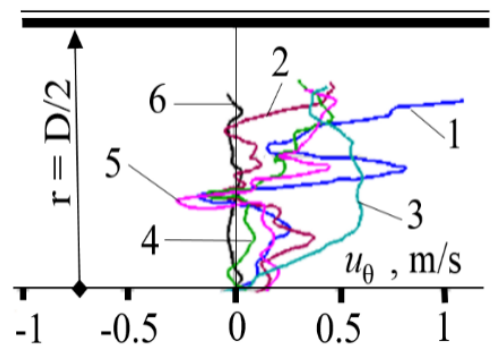

$b$

Fig.5. Six-layered flow. Profiles: $a$-axial $u_{x}$ and $b$-azimuthal $u_{\theta}$ flow velocities. $1-0.2 r, 2-1.3 r$, $3-1.9 r, 4-3.4 r, 5-5.2 r, 6-8.1-8.8 r$

that maintains the balance of volume flow. There is a reverse paraxial flow with interaction of two layers in the section of up to 4 radii of the length of the active interaction area while an increase in the number of coaxial oppositely swirled layers (from 2 to 6) reduces to an extent the length of the section with reverse flow. Comparison of the type of experimental diagrams with the diagrams obtained by calculation (analytical method) suggests that they coincide in nature [18], despite the fact that the computed diagrams have been obtained for the laminar flow regime.

An increase in the number of layers leads to faster completion of the interaction process, after which the flow, having lost energy, changes its structure and enters the conventional 
longitudinal-axial turbulent flow regime with Reynolds number $R e=3.8 \times 10^{4}$ and velocity distribution according to the logarithmic law. An exit to such a regime with two-layered flow occurs at a length of about $18 R$, with four-layered one - $13 R$, with six-layered one (8-9) $R$.

In general, the nature of distribution (profiles in Fig. 3-5, a) of axial velocities $u_{x}$ along the length $l$ of the interaction area of the layers indicates that the complicated, alternating longitudinal flow retains a rather high degree of symmetry with respect to the pipe axis along the entire length of the interaction of the layers of the three tested models.

Transformation of azimuthal velocity $u_{\theta}$ profiles along the length of the active area is shown in Fig.3-5, b. The analysis of the obtained results shows that the flow with approximately equal moments of momentum of the oppositely swirled coaxial layers quickly transforms into longitudinal-axial flow, that is, without vortex. This can be observed on the profiles shown in Fig. 3-5, b, where the active zone, the zone of intense viscous diffusion of the circulation of the interacting layers, lies in the range of 8-18 pipe radii. The fact of intensive diffusion of circulation of interacting layers is obvious; it does not require detailed explanations, because it is determined by the mutual suppression of the circulation of coaxial oppositely rotating layers. The intensity of mutual suppression of circulation increases with increasing number of interacting layers. The azimuthal velocity profiles presented in [19], obtained by calculation for the value of Reynolds numbers $R e=500$ (laminar flow) have a similar appearance. However, since the flow regime is laminar, the process of viscous diffusion of circulation of the interacting layers terminates here at the 40th radius of the interaction area.

In this regard, the following should be noted. In a number of previous papers [20, 21, $22,23]$, it was shown that when calculating turbulent circulation-longitudinal fluid flows, good convergence with experimental data is obtained using a turbulent analogue of the Reynolds number calculated from the vortex viscosity of a turbulent medium.

The values of the radial velocities $u_{r}$ of the flows for all the experiments performed in general are significantly lower than the axial and azimuthal ones. This is especially observed in the sections remote from the beginning of the interaction area of the layers. Already at a distance of 1.5 fractions of the radius from the beginning of the interaction area the values of the radial velocities are $0.1-0.3 \mathrm{~m} / \mathrm{s}$.

Azimuthal velocities form radial liquid mass flows directed towards the axis of the channel (negative radial velocities). Thus, the flow is not available in all the sections. Here it is necessary to take into account that at the beginning of the active zone we observe a substantial return flow in the area of the axis in both the two-layered and multi-layered swirled flow. As we see, it forms an area with recirculation motion around itself, in which just at the beginning of the active zone an upward fluid flow from the axis towards the pipe walls will be observed. This can be characterized as the beginning of transformation of the flow to the longitudinal-axial one with logarithmic velocity distribution featuring the developed turbulent flow. The experiments show that the radial velocities become close to zero long before the end of the area of interaction of the layers, therefore, they can, in some cases, be neglected The return paraxial flow noted above in the area of active interaction of the layers forms a recirculation movement around itself with no mass exchange between it and transit flow streamlining it and the mass inside the recirculation bubble does no go downstream. In accordance with the property of the streamlines, the tangents to which at any point coincide with the direction of the velocity vectors of elementary fluid particles located on them at a given time moment and in steady-state flows coincide with the trajectories of the particles, it can be assumed that the described recirculation area is a circular toroidal $\theta$ vortex with closed elliptic streamlines $\psi$ and is limited by isoline, having a zero value $(\psi=$ $0)$. 
The distributions obtained in Figs. 6 and 7 show the presence of several recirculation zones in the interaction area of oppositely rotating layers. Moreover, the paraxial recirculation zones, which are toroidal $\theta$-vortices, both in the two-layered (Fig. 6) and in the four-layered (Fig. 7) flows are highly developed in the radial and axial directions. With a two-layered flow the zone of recirculation movement is significantly more stretched along $x$-axis than in the four-layered flow, but in the latter it is more developed along the radius. In the twolayered counter-vortex flow one can see at least one more area with recirculation movement in the immediate vicinity of the swirling device located above the axial recirculation zone. Such a cellular structure is characteristic for the areas of decay of circulation-longitudinal flow layers, for example, in the form of the so-called bubble form [24, 25, 26]. In comparison with the two-layer, the multilayered flow has an even more complicated cellular structure, which contributes to its even more intense decay.
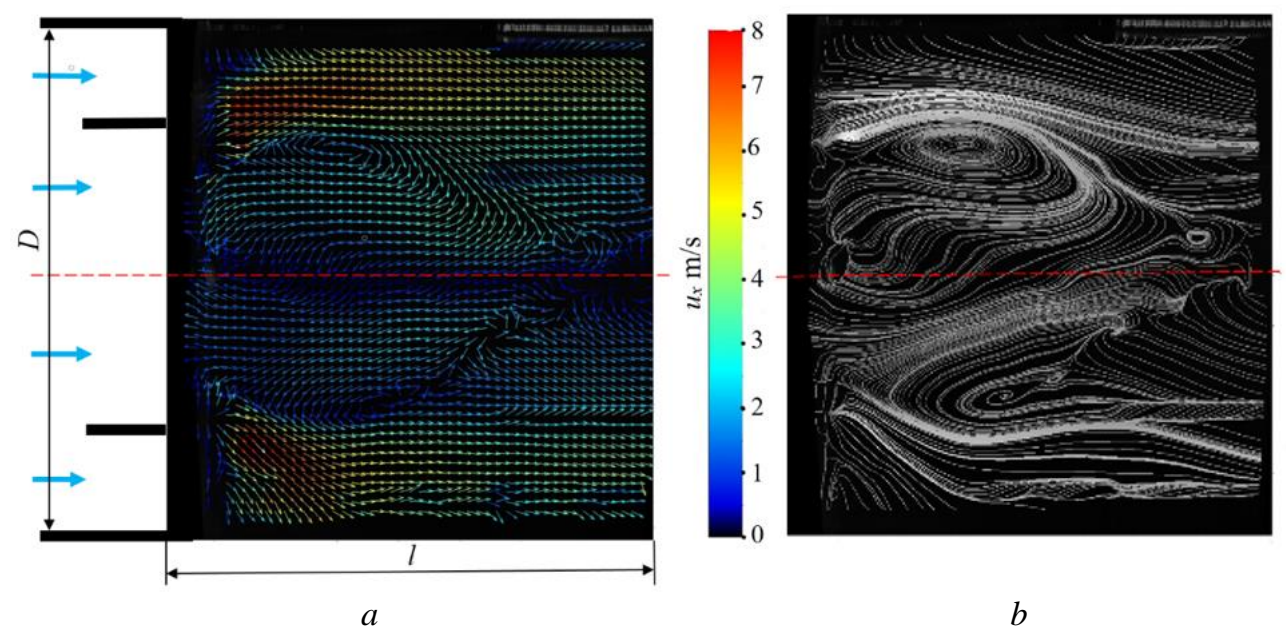

Fig. 6. Two-layered flow. $a$ - field of velocity vectors, $b$ - streamlines
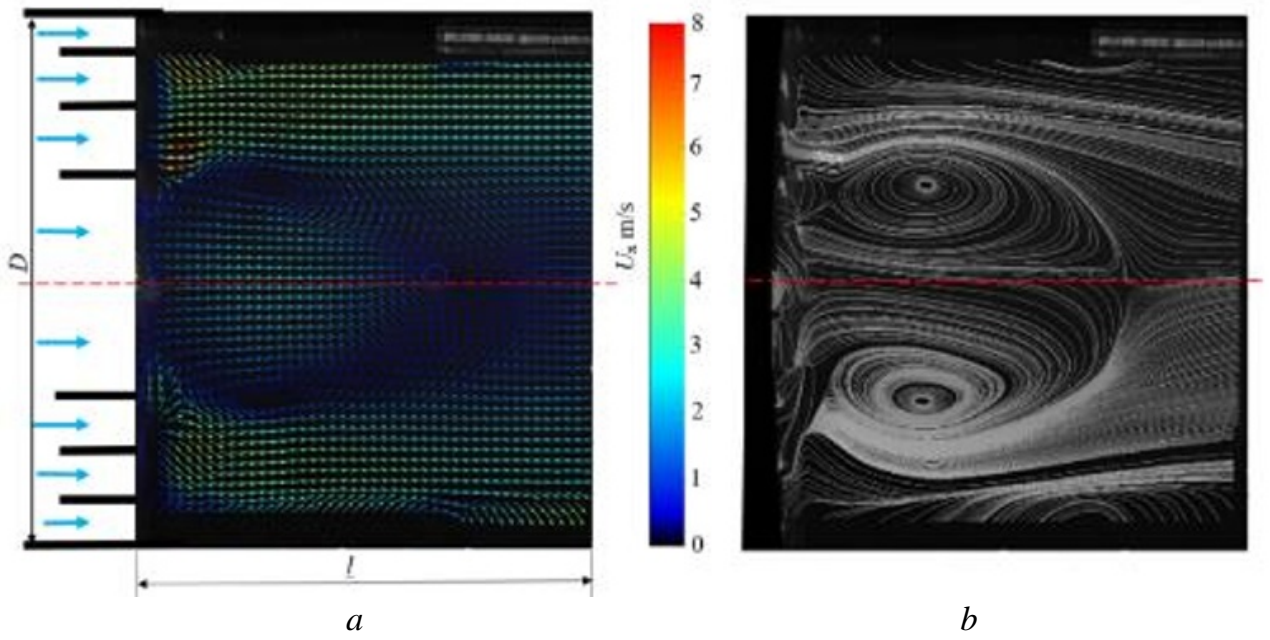

Fig. 7. Four-layered flow. a - field of velocity vectors, $b$ - streamlines 


\section{Conclusion}

Based on the above, we can formulate the main features of the kinematics of oppositely rotating multilayer flows:

- the studies of complicated flows carried out by the physical method using special laser systems made it possible to penetrate more deeply into the structure of layered swirled flows with the number of layers two, four and six;

- the obtained profiles of the axial velocity $u_{x}$ components showed that in the area of interaction of the swirled layers there is a zone with negative values of the flow velocities directed towards the swirling device;

- the circulation of the flow in the interaction zone decreases in the direction of flow and degenerates completely. The zero value of circulation is observed: for a two-layer flow at a length of $18 R$, for a four-layer flow - $13 R$, for a six-layer flow - (8-9) $R$. These results indicate an increase in the circulation diffusion along the length $l$ with an increase in the interacting layers. This indicates an increase in the degree of the initial energy dissipation of the entire flow;

- with decrease of circulation to zero the transition to conventional longitudinal-axial turbulent flow with a logarithmic velocity distribution over the cross section of the flow conductor. Therefore, the length of the active interaction area $l$ is determined by the distance from the swirler to the cross section with a logarithmic velocity distribution.

- spatial fixation of the active interaction areas for multilayer swirled systems showed that for all the flows there is a recirculation zone, which begins immediately downstream of the swirling device. It is clearly visible in the experimentally obtained streamlines. The recirculation zone is limited by the area with zero streamline with the liquid mass not going beyond this boundary and remaining in it throughout the entire length of the flow existence in the steady-state flow regime. The transit flow, which determines the flow rate, streamlines the recirculation zone, closing it.

\section{References}

1. B. Sazhin, M. Sazhina, M. Aparushkina et al., News of the universities. Technology of the textile industru 1(343) 135-138 (2013)

2. A. Ianiro, K. Lynch, D. Violato, Cardone G, J. of Fluid Mechanics 843 180-210 (2018)

3. A. Zuikov, Vestnik MGSU (Proceedings of the Moskow State University of Civil Engineer-ing) 5 150-161 (2013)

4. T. Parra-Santos, R. Perez, R. Szasz, A. Gutkowski, F. Castro EPJ Web of Confereces, 11402087 (2016)

5. F. Muhammad, I. Muhammad, B. Niaz, M. Riaz, U. Muftooh, W. Sajjad, A. Tufail, Results in Physics 9 1250-1255 (2018)

6. S. Shehzad, A. Alsaedi, T. Hayat, M. Alhuthali J. of the Taiwan Institute of Chemical Engineers 45 (3) 787-794 (2014)

7. F. Lemperiere, Engineering 3, 144-149 (2017)

8. Hydraulic calculations waterworks intakes. Reference manual. (Moscow, Publ. Ener goatomizdat. 233-248 1988)

9. G. Orekhov. Ecology of the urbanized territories. 2 88-93 (2008)

10. A. Zuikov, G. Orekhov, V. Volshanik, Vestnik MGSU, 5 150-161 (2013)

11. V. Karelin, A. Mordasov, A. Zuikov, V. Volshanik, Numerical methods of experimental studies of the characteristics of swirling flow. Proceedings of the Symposium MIGA, Belgrade, Yugoslavia (1990) 
12. V. Akhmetov, V. Volshanic, A. Zuikov, G. Orekhov, Simulation and calculation of counter-rotating flows. Monograph. (Moscow State University of Civil Engineering Publ., 2012)

13. G. Orekhov, XXI International Scientific Conference on Advanced in Civil Engin. FORM2018, MGSU, april 25-27. IOP Conference Series: Materials Science and Engin. 365042023 (2018)

14. V. Volshanik, A. Zuikov, G. Orekhov, P. Churin. Power Techn. and Engin. 4 51-56 (2013); 5 32-40 (2013)

15. V. Volshanik, A. Zuikov, G. Orekhov, Uranzaya Bayaraa, The public scientific j. Ecology of urban areas, 1 23-28 (2015)

16. S. Dehdar-Behbahani, A. Parsaie, Alexandria Engineer. J. 55 467-473 (2016)

17. A. Zujkov, Hydrodynamics of circulation currents. (ASV 216 pp. 2010)

18. V. Akhmetov, V. Volshanik, A. Zuikov, G. Orekhov, Modelling and calculation counter vortex currents (Edited by A. Zuikov. Moskov, Publ. MGSU 252 p. 2012)

19. Z. Mohammad, M. Rashid Sarkar, International J. of Engineering Materials and Manuf. 3(3) 122-133 (2018)

20. V. Akhmetov, V. Shkadov, Numerical modeling of viscous vortex flows for technical applications. (Moskov, Publ. ASV, 2009)

21. A. Zuikov, V. Volshanik, Analytical study of the flow structure of a viscous incompressible fluid in a cylindrical tube, (Moskov, Publ. ASV, 2001)

22.Z. Zhang, R. Liao, J. Liu, Advances in Mechanical Engineering 10 (9) 1-11 (2018)

23.Z. Ruan, W. Baars, M. Abbassi, N. Hutchin, I. Marusic, 21st Australasian Fluid Mechanics Conference Adelaide, Australia, 10-13 December (2018)

24. J. Delery, Progr. Ferospace Sei. 30 1-59 (1994)

25. J. Faler, S. Leibovich, J. Fluid Mech. 86 (2) 313-335 (1978)

26. W. Grabowski, S. Berger, J. Fluid Mech. 75 (3) 525-544 (1976) 